\title{
APPLICATION DE L'ANALYSE THERMIQUE DIFFÉRENTIELLE A L'ÉTUDE DES VERRES
}

\author{
M. PROD'HOMME
}

\author{
C. N. R. S., Institut du Verre, 34, rue Michel-Ange, 75016 Paris, France
}

\begin{abstract}
Résumé. - L'analyse thermique différentielle est très souvent utilisée dans le domaine de la recherche et de la technologie verrières. L'application de cette méthode simple au verre s'est développée grâce aux travaux de Tool et ses collaborateurs qui étudièrent de cette façon le comportement du verre aux températures du domaine de transformation.

Dans cet article sont présentés plusieurs exemples de l'emploi de cette méthode pour l'étude des effets thermiques produits, soit pendant la fabrication du verre, soit au cours du chauffage dans le domaine de transformation, ou encore pendant les changements de phase apparaissant dans certains verres.

Abstract. - Differential thermal analysis is very often used in glass research and technology. This simple method was developed by Tool and his coworkers who studied the behaviour of glass at temperatures within the annealing range.

In this paper, a survey is presented of the work that has been carried out on the thermal effects observed in the transformation range or when phase changes occur in certain glasses.
\end{abstract}

1. Introduction. - L'analyse thermique différentielle a connu ses premières applications au verre en 1916 pour l'étude du refroidissement d'un verre à glace [1] et elle fut utilisée plus largement quelques années plus tard dans les travaux de Tool et ses collaborateurs [2] sur les effets thermiques apparaissant dans le domaine de transformation. Depuis, l'analyse thermique différentielle a connu une très large utilisation dans la recherche et la technologie verrières. Après un bref rappel du principe de la méthode, nous considérerons successivement les applications suivantes :

1) étude des réactions chimiques apparaissant pendant la préparation du verre,

2) étude des phénomènes thermiques du domaine de transformation,

3) étude des changements de phase : démixtion, dévitrification.

2. Méthode. - La méthode d'A. T. D. est très simple. Elle consiste à chauffer dans les conditions identiques, l'échantillon à mesurer dont les propriétés thermiques sont inconnues et un autre échantillon de forme identique ou substance de référence qui ne présente pas d'anomalie thermique dans le domaine de température choisi, ou dont on connaît la chaleur spécifique.

Les différences entre les propriétés thermiques des divers échantillons se traduisent par des différences de températures entre ceux-ci. Les différences de températures sont mesurées par un thermocouple différentiel dont les deux soudures sont situées respectivement dans chaque échantillon. La température de l'échantillon à mesurer est déterminée par un autre thermocouple, et on peut suivre ainsi la variation de la différence de température entre les deux échantillons en fonction de la température. Cette variation met en évidence les caractéristiques thermiques du corps étudié.

3. Appareillage. - Les appareils utilisés pour l'A. T. D. peuvent se répartir en plusieurs catégories [3] :

- les appareils qui permettent de faire seulement une analyse qualitative : ils sont de construction simple, ont une très bonne résolution et une bonne précision de température ;

- les appareils qui permettent de faire une analyse quantitative : ils donnent des résultats très reproductibles et la possibilité de relier la hauteur ou l'aire du pic à la quantité de substance réagissant ;

- les appareils qui permettent de faire des mesures calorimétriques. Quoique plus complexes que les précédents, ils sont basés sur le même principe d'une mesure différentielle.

4. Applications. - 4.1 RÉACTIONS PENDANT LE PROCESSUS DE FABRICATION DU VERRE. - Afin de comprendre comment le liquide verrogène se forme, il est nécessaire d'étudier les réactions chimiques qui apparaissent au cours du chauffage du mélange.

L'A. T. D. peut être effectuée par mesure directe sur le mélange initial, mais on peut également effectuer 
l'A.T.D. sur un mélange ayant été préalablement chauffé à une certaine température, puis refroidi.

Etant donné la grande complexité des réactions qui se produisent et la variété des composés intermédiaires qui se forment dans le cas d'un mélange complexe, de nombreux auteurs ont préféré opérer d'abord sur des mélanges simples.

Les réactions entre les constituants ont été étudiées en fonction de leur granulométrie et de leur concentration.

Wilburn et ses collaborateurs [4] ainsi ont montré que la dimension des grains de chaque substance avait une influence marquée sur le cours de la réaction chimique. En général, à partir de grains de dimensions plus faibles, on obtient des fontes qui peuvent être refroidies pour former un verre à des températures plus faibles.

Oldfield [5] a étudié les réactions dans le système $\mathrm{Na}_{2} \mathrm{CO}_{3}-\mathrm{SiO}_{2}$ en fonction de la composition, les deux constituants ayant des dimensions de grains comprises entre 76 et $124 \mu \mathrm{m}$ (Fig. 1).

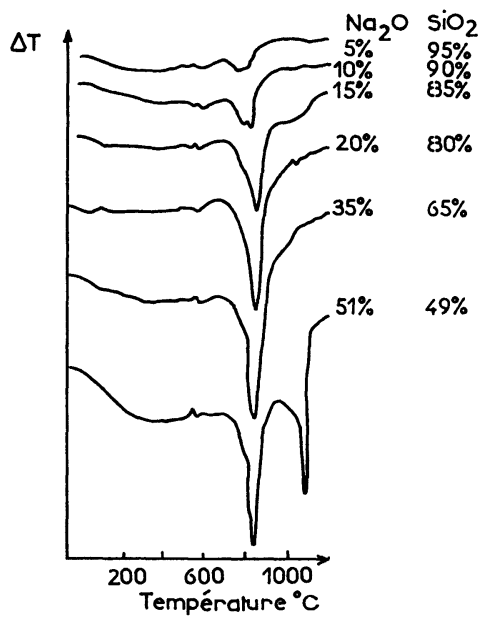

Fig. 1. - Courbes d'A. T. D. pour les mélanges $\mathrm{Na}_{2} \mathrm{O}-\mathrm{SiO}_{2}$ d'après Oldfield (1958).

Les courbes d'A. T. D., pour les mélanges dans lesquels la silice est le composant majeur, montrent un pic exothermique entre $600^{\circ} \mathrm{C}$ et $750^{\circ} \mathrm{C}$ suivi de deux pics endothermiques à $780^{\circ} \mathrm{C}$ et $830^{\circ} \mathrm{C}$; lorsque le pourcentage de $\mathrm{Na}_{2} \mathrm{CO}_{3}$ augmente, ces deux derniers pics coalescent. Le pic exothermique dans la région $600-750^{\circ} \mathrm{C}$ est attribué à la réaction initiale entre le carbonate de sodium et la silice. Les pics endothermiques à $780^{\circ} \mathrm{C}$ et $830^{\circ} \mathrm{C}$ sont dus respectivement à la fusion eutectique entre le bisilicate de sodium et la silice et à la fusion eutectique entre le bisilicate et le métasilicate de sodium. Pour le mélange $51 \% \mathrm{Na}_{2} \mathrm{O}$, $49 \% \mathrm{SiO}_{2}$, on trouve 2 pics à $850^{\circ}$ et $1094{ }^{\circ} \mathrm{C}$. Oldfield a suggéré que ceux-ci étaient dus à une réaction entre le carbonate de sodium et la silice pour former du bisilicate de sodium qui fond approximativement à environ $860^{\circ} \mathrm{C}$, suivie par une réaction entre le carbonate de sodium, l'oxyde de sodium et la silice pour former du métasilicate de sodium.

Wilburn et ses collaborateurs [6] ont étudié les réactions dans un mélange de composition analogue au verre à vitre composé de sable, carbonate de sodium, dolomie et de chaux. A partir des réactions binaires possibles, ils ont conclu que lorsque l'on chauffe le mélange, le carbonate de sodium réagit avec le calcaire et la dolomie pour former des carbonates doubles de sodium et de calcium à $500^{\circ} \mathrm{C}$ environ. Deux de ces carbonates doubles fondent sous forme d'eutectique à $750^{\circ}$ et $780^{\circ} \mathrm{C}$ fournissant une phase liquide qui réagit rapidement avec le carbonate de sodium et la silice à $850^{\circ} \mathrm{C}$. La première phase liquide qui puisse être trempée sous forme de verre apparaît à $900^{\circ} \mathrm{C}$. La silice se dissout alors lentement dans ce liquide, le taux de dissolution atteignant $86 \%$ à $1100^{\circ} \mathrm{C}$.

\subsection{PHÉNOMÈNES THERMIQUES APPARAISSANT DANS} LE DOMAINE DE TRANSFORMATION. - L'étude par A. T. D. des modifications structurales du verre apparaissant dans le domaine de transformation a été, comme nous l'avons dit, la première application au verre de cette méthode.

Tool et ses collaborateurs [2] firent une étude systématique des effets thermiques apparaissant dans le domaine de transformation et surtout de l'influence de l'état du verre sur ces effets. Sur le thermogramme d'un verre, la différence de température entre le verre et le corps de référence est d'abord constante, puis dans le domaine de transformation on observe dans le cas où le verre est trempé, un pic exothermique suivi d'un effet endothermique. Si le verre est bien recuit, l'effet exothermique est nul, tandis que l'effet endothermique est très grand. Entre ces deux états, les thermogrammes peuvent présenter toutes les situations intermédiaires.

Depuis les travaux de Tool, de nombreux chercheurs ont utilisé cette méthode par exemple pour déterminer les températures caractéristiques des verres ou pour interpréter les changements de structure et de propriétés du verre dans le domaine de transformation.

Lorsqu'un liquide surfondu peut être refroidi jusqu'à la zone de température où le verre se forme, les courbes représentant les variations des propriétés telles que le volume spécifique ou l'enthalpie, subissent un rapide changement de leur pente.

On définit généralement une température $T_{\mathrm{g}}$ appelée température moyenne de transformation qui correspond au passage de l'état liquide surfondu à l'état vitreux. Cette température $T_{\mathrm{g}}$ varie avec la vitesse de refroidissement. Ce résultat s'explique si on considère la variation d'enthalpie d'un liquide surfondu [7]. Au cours d'un refroidissement rapide de la température $T_{1}$ à la température $T_{2}$ (Fig. 2), le système prend la valeur $H_{0}$ et ne se trouve plus en équilibre thermodynamique. Par un traitement isotherme à la température $T_{2}$, ou recuisson, il évolue vers l'état d'équilibre métastable du liquide surfondu correspondant à cette température. Les verres trempés, obtenus par un 


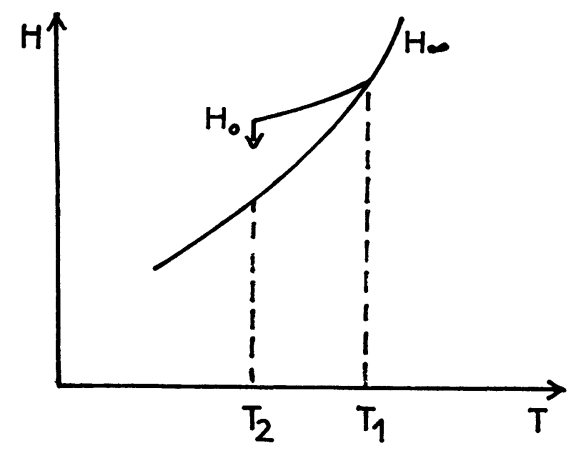

Fig. 2. - Variation de l'enthalpie du liquide surfondu au cours du refroidissement.

refroidissement rapide, ont donc une plus grande enthalpie que les verres recuits.

Au cours du chauffage d'un verre (Fig. 3), l'évolution vers l'équilibre est très lente à basse température, et le verre ne subit pas tout d'abord de transformation. Ensuite, dès que la vitesse d'évolution vers l'équilibre

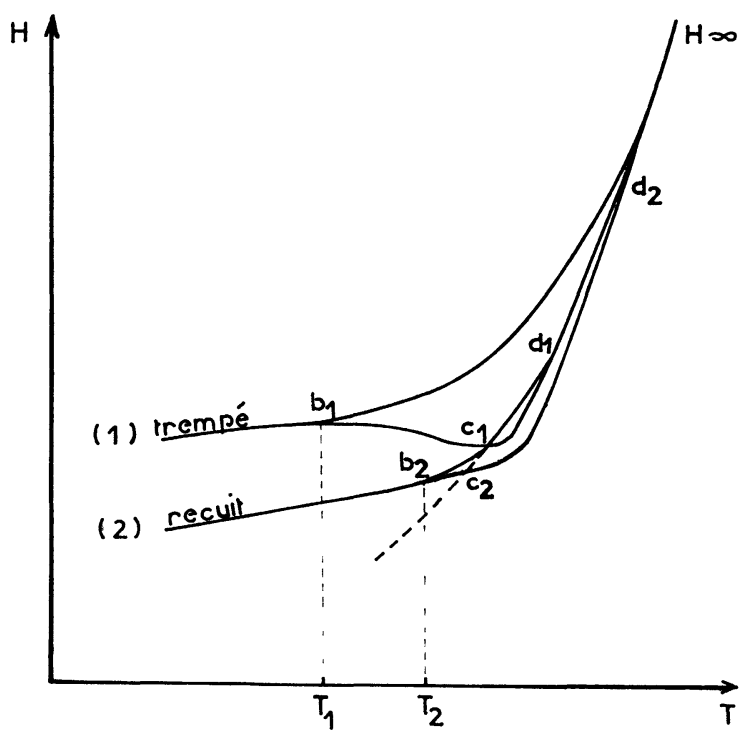

Fig. 3. - Variation de l'enthalpie d'un verre trempé et d'un verre recuit au cours du chauffage.

est suffisante, l'enthalpie diminue. L'effet exothermique qui en résulte, débute pour le verre trempé à une température $T_{1}$ inférieure à celle du verre recuit $T_{2}$ et il est plus important pour le verre trempé que pour le verre recuit (portions de courbe $b_{1} c_{1}$ et $b_{2} c_{2}$ ). Puis le processus de mise en équilibre se fait avec augmentation d'enthalpie et l'effet endothermique résultant est plus grand pour le verre recuit que pour le verre trempé (portions de courbe $c_{2} d_{2}$ et $c_{1} d_{1}$ ). L'effet exothermique qui débute à basse température est lent, tandis que l'effet endothermique qui le suit est rapide, car au fur et à mesure que la température s'élève, la viscosité devient plus faible et l'évolution vers l'équilibre est alors plus rapide.
Ainsi un verre trempé présente un effet exothermique lent et assez marqué suivi d'un effet endothermique faible, tandis qu'un verre recuit présente un effet endothermique brutal précédé d'un effet exothermique faible.

Du point de vue pratique, le début et le minimum du pic endothermique sont très souvent utilisés pour déterminer deux températures caractéristiques du verre. La température du début du pic correspond à la température moyenne de transformation $T_{\mathrm{g}}$ $\left(\log \eta_{\text {poises }}=13,3\right)$ et la température du minimum du pic correspond à la température de déformation $M_{\mathrm{g}}$ $\left(\log \eta_{\text {poises }}=11\right)$. Pour la détermination de ces températures caractéristiques $T_{\mathrm{g}}$ et $M_{\mathrm{g}}$, la vitesse de chauffage et l'état structural du verre (trempé ou recuit) doivent donc être précisés. Par exemple, pour un verre d'optique crown, les températures $T_{\mathrm{g}}$ et $M_{\mathrm{g}}$ augmentent de $20^{\circ}$ environ si la vitesse de chauffage augmente de $3 \% / \mathrm{min}$. à $20 \% \mathrm{~min}$. [8].

Nous pouvons voir sur la figure 4, les thermogrammes de ce verre crown recuit optique, du même verre

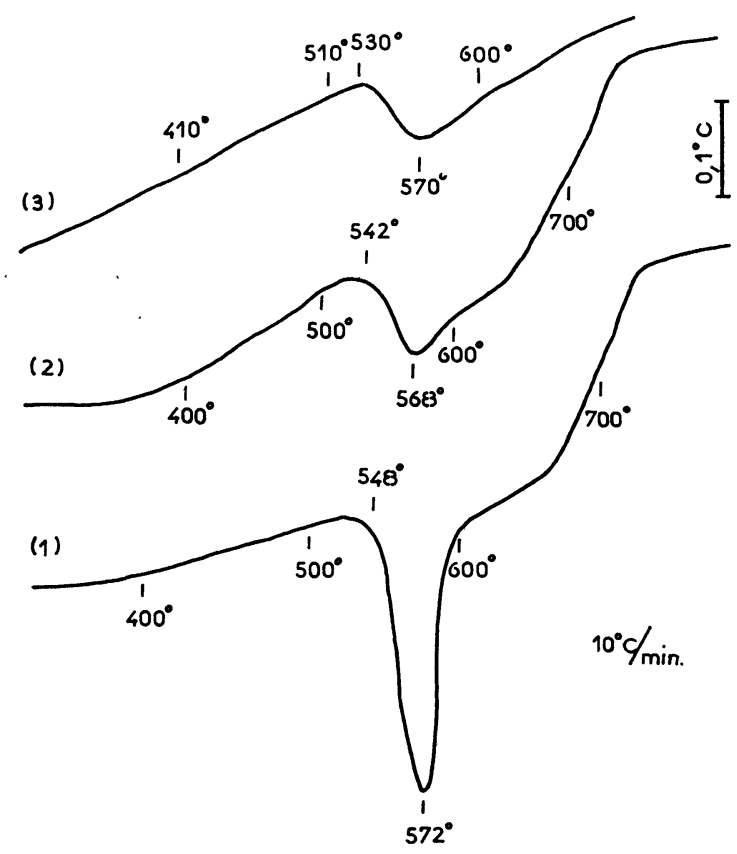

Fig. 4. - Thermogrammes d'échantillons d'un verre crown ayant subi un recuit optique (1), une trempe industrielle (2), ou sous forme d'un faisceau de fibres de $60 \mu \mathrm{m}$ environ (3) d'après Lambert-Dusollier (1975).

ayant subi une trempe industrielle c'est-à-dire refroidi rapidement à partir de la température de ramollissement et enfin du même verre sous forme de fibre ayant donc subi une trempe encore plus sévère puisque refroidi très rapidement à partir du liquide [8].

4.3 Etude des Changements DE PHASE. 4.3.1 Démixtion. - De nombreuses compositions de mélanges binaires ou ternaires ne restent pas au cours 
du refroidissement sous la forme d'une phase vitreuse unique, mais se figent dans un état amorphe complexe contenant plusieurs phases vitreuses de compositions différentes. Cette démixtion peut apparaître de deux façons différentes soit pas nucléation qui produit des phases discontinues soit par décomposition spinodale conduisant à des phases interconnectées. Dans ces matériaux, chaque phase doit se transformer de l'état liquide surfondu à l'état vitreux dans son propre domaine de transformation. Donc un verre à deux phases doit présenter deux points de transformation différents. L'A. T. D. permet de connaître les valeurs des températures $T_{\mathbf{g}}$ des différentes phases et aider à déterminer les directions des conodes.

Les premières études dans ce domaine sont également dues à Tool [9] qui en 1948 étudia l'influence du traitement thermique sur la structure des verres borosilicatés à haute teneur en silice. Il signala la présence de contraintes mécaniques provoquées par la différence de dilatation thermique des deux phases séparées : l'une riche en silice, l'autre riche en borate de soude. Les thermogrammes de ces verres présentaient deux effets endothermiques à $580^{\circ}$ et $650^{\circ} \mathrm{C}$.

Broukal [10] ainsi que Kühne et Skatulla (1959) [11] montrèrent également que les thermogrammes des verres démixés indiquaient plusieurs effets endothermiques. Par exemple pour un verre de composition molaire $64,4 \% \mathrm{SiO}_{2}, 30 \% \mathrm{~B}_{2} \mathrm{O}_{3}$ et $5,6 \% \mathrm{Na}_{2} \mathrm{O}$, le thermogramme peut présenter trois effets endothermiques. Kühne et Skatulla indiquèrent que le premier correspondait à l'acide borique vitreux qui possède le point de fusion le plus bas. Le deuxième serait dû à une phase borate riche en alcali. Le troisième effet, observé uniquement pour un verre maintenu en température et par conséquent siège d'une forte démixtion, est dû très probablement à la phase riche en silice. En raison d'une certaine solubilité résiduelle des constituants, ces températures de transformation sont différentes de celles obtenues avec le verre de $\mathrm{B}_{2} \mathrm{O}_{3}$ pur ou avec le verre de silice.

Pour l'étude de la séparation de phase des systèmes binaires, il est suffisant de connaître la limite de la région de séparation de phase. Dans les systèmes ternaires il faut également connaitre les directions des conodes. La composition des phases est déterminée en général par analyse chimique mais dans certains cas, par exemple si les domaines de phase sont de très petites dimensions - de l'ordre du micron - cette analyse est impossible. Plusieurs méthodes ont alors été proposées pour déterminer les conodes. Mazurin, Streltsina et Totesh $[12,13]$ suggérèrent que les lignes d'égales propriétés (par exemple les isothermes de transition vitreuse) d'un verre à phases séparées devaient coïncider avec les conodes. Il faut pour cela que la propriété dépende seulement de la composition d'une des phases et non de la quantité de la phase dans le verre, ce qui est le cas de $T_{\mathrm{g}}$. Ils déterminèrent par cette méthode les conodes dans la région de séparation de phase des borosilicates de sodium qui coïnci- daient bien avec celles déterminées par d'autres méthodes. La validité de cette méthode a également été confirmée par Zarzycki et Naudin qui déterminèrent les conodes dans le système ternaire $\mathrm{B}_{2} \mathrm{O}_{3}-\mathrm{PbO}-\mathrm{Al}_{2} \mathrm{O}_{3}$ [14].

Sur la figure 5, nous présentons les thermogrammes que nous avons obtenus [15] pour deux borosilicates de $\mathrm{Na}$ de compositions différentes et sujets tous les deux à une démixtion en deux phases : l'une enrichie en $\mathrm{B}_{2} \mathrm{O}_{3}$ et l'autre en $\mathrm{SiO}_{2}$.

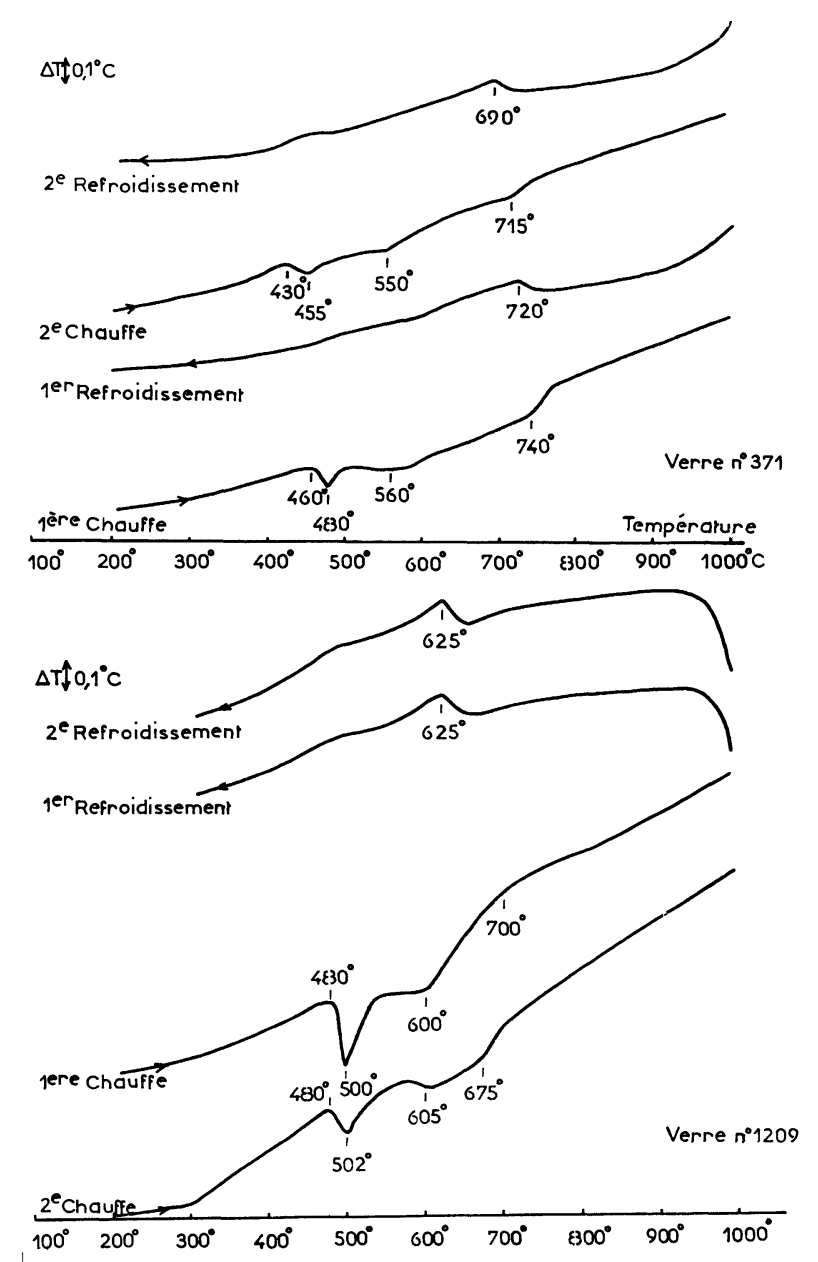

FIG. 5. - Thermogrammes de deux borosilicates de sodium présentant une séparation de phases.

Verre 371. - $\mathrm{SiO}_{2}: 68, \mathrm{~B}_{2} \mathrm{O}_{3}: 27, \mathrm{Na}_{2} \mathrm{O}: 5(\% \mathrm{~mol})$. Les températures $T_{\mathrm{g}}$ des deux phases se situent à environ $480^{\circ}$ et $560^{\circ} \mathrm{C}$. Pour ce verre dont la structure est interconnectée, les deux phases sont continues et les deux effets endothermiques ont à peu près la même importance.

Verre 1209. - $\mathrm{SiO}_{2}: 60, \mathrm{~B}_{2} \mathrm{O}_{3}: 30, \mathrm{Na}_{2} \mathrm{O}: 10$ (\% mol). Les températures de transformation $T_{\mathrm{g}}$ des deux phases sont respectivement de $500^{\circ}$ et $600^{\circ} \mathrm{C}$. Les deux phases de ce verre sont par contre discontinues et le premier effet endothermique dû à la phase la plus fluide riche en $\mathrm{B}_{2} \mathrm{O}_{3}$ est le plus important car cette phase constitue la matrice vitreuse dans laquelle 
se trouve la deuxième phase riche en $\mathrm{SiO}_{2}$ sous forme d'îlots discontinus.

4.3.2 Dévitrification. - Lors de la dévitrification d'un verre, il se produit un effet exothermique car le verre passe d'un état d'équilibre métastable à un état stable et son énergie libre diminue.

Le domaine de température où cet effet exothermique se manifeste sur un thermogramme d'A.T.D. pendant la dévitrification, et plus particulièrement la température du maximum du pic $T_{\mathrm{m}}$, dépendent de nombreux facteurs tels que la quantité de matériau utilisé, sa granulométrie, la vitesse de chauffage [16].

Ainsi sur les thermogrammes d'un verre silicosodocalcique de composition (\% poids): $\mathrm{SiO}_{2}: 70$, $\mathrm{CaO}: 13, \mathrm{Na}_{2} \mathrm{O}: 17$, enregistrés à une vitesse de chauffage de $10^{\circ} \mathrm{C} / \mathrm{min}$. (Fig. 6), on peut observer un

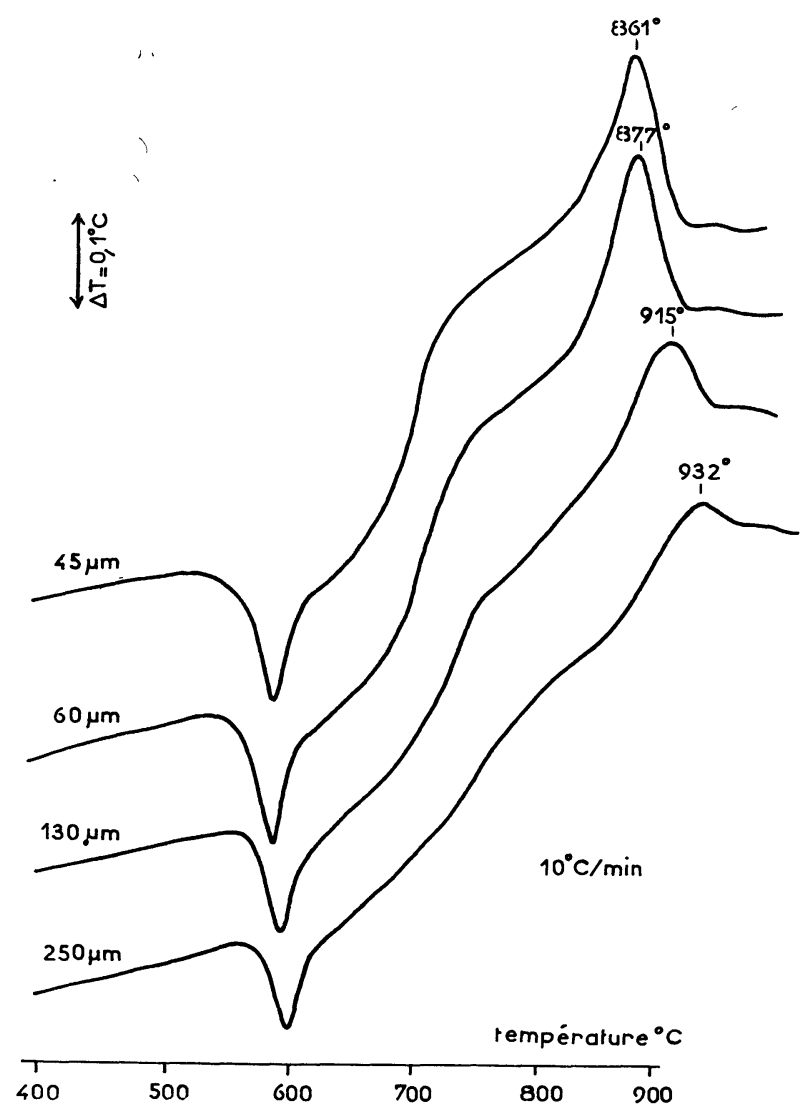

FIG. 6. - Thermogrammes d'échantillons de verre silicosodocalcique de granulométrie différente : $45 \mu \mathrm{m}, 60 \mu \mathrm{m}, 130 \mu \mathrm{m}$ et $250 \mu \mathrm{m}$.

déplacement de la température $T_{\mathrm{m}}$ du maximum du pic exothermique de 860 à $932^{\circ} \mathrm{C}$ lorsque la dimension des grains croît de 45 à $250 \mu \mathrm{m}$. Sur les thermogrammes de ce verre de granulométrie moyenne $60 \mu \mathrm{m}$ réalisés à différentes vitesses de chauffage, on constate que la température $T_{\mathrm{m}}$ croît de $828^{\circ}$ à $914^{\circ} \mathrm{C}$ lorsque la vitesse de chauffage augmente de $3 \% / \mathrm{min}$. à $21 \% \mathrm{~min}$. Si on suppose, comme l'a fait Kissinger [17], que la vitesse de réaction est maximum à la température $T_{\mathrm{m}}$, on peut déterminer l'énergie d'activation de la réaction à partir des thermogrammes réalisés à différentes vitesses de chauffage. La vitesse de réaction s'écrit :

$$
\frac{\mathrm{d} x}{\mathrm{~d} t}=A(1-x) \mathrm{e}^{-E / R T}
$$

où $x$ est la fraction de matière cristallisée, $A$ le terme préexponentiel ou facteur de fréquence et $E$ l'énergie d'activation de la réaction.

En dérivant cette équation par rapport au temps et en admettant qu'à la température $T_{\mathrm{m}}$, la vitesse de réaction est maximale, on obtient :

$$
\frac{E}{R T_{\mathrm{m}}^{2}} \frac{\mathrm{d} T}{\mathrm{~d} t}=A \mathrm{e}^{-E / R T_{\mathrm{m}}}
$$

On vérifie que $\operatorname{Ln}\left(\varphi / T_{\mathrm{m}}^{2}\right)$ est bien linéaire en fonction de $1 / T_{\mathrm{m}}(\varphi=\mathrm{d} T / \mathrm{d} t$ est la vitesse de chauffage). La pente de la courbe permet de calculer l'énergie d'activation de la réaction $E$ et l'ordonnée à l'origine le facteur de fréquence $A$.

En faisant cette étude pour différentes granulométries, on trouve que l'énergie d'activation est un peu plus élevée lorsque la dimension des grains est plus faible. Ceci signifie que la vitesse de dévitrification varie alors plus rapidement en fonction de la température.

On trouve également que le facteur de fréquence est beaucoup plus important lorsque la granulométrie des échantillons est plus faible, c'est-à-dire que la probabilité qu'une molécule d'énergie $\mathrm{E}$ participe à la réaction est alors plus grande.

Les thermogrammes permettent également de comparer les quantités de chaleur mises en jeu pendant la dévitrification en mesurant la hauteur et la surface des pics.

En comparant l'aire des pics pour des échantillons de granulométries différentes, on constate que pour des grains plus petits, les quantités de chaleur dégagées pendant la dévitrification sont plus élevées. On pouvait s'attendre à ce résultat car nous avons vu qu'un plus grand nombre de molécules participait alors à la réaction.

Récemment, Briggs et Carruthers [18] ont également utilisé l'A. T. D. pour obtenir des informations cinétiques quantitatives sur la vitesse de croissance cristalline des verres. Ils relièrent quantitativement la forme de l'effet exothermique obtenu expérimentalement à la cinétique de la cristallisation et trouvèrent ainsi la variation de la vitesse de croissance cristalline en fonction de la température pour d'autres compositions de verre.

Nous signalerons aussi une technique dérivée de celle de l'A. T. D. qui est l'analyse thermique bidifférentielle. Cette méthode consiste à employer comme référence une substance presque identique à celle de l'échantillon étudié. Cette façon d'opérer permet de déceler de faibles différences de comportement, mais son utilisation est limitée à des cas très particuliers : 
déplacements d'accidents thermiques dus à un traitement préliminaire, ou bien à un très léger changement de la composition; comparaison de minéraux semblables mais d'origine différente [16].

5. Conclusion. - Cette revue des applications de l'analyse thermique différentielle à l'étude de phénomènes intéressant la recherche et la technologie du verre montre bien les possibilités offertes par cette méthode dans ce domaine. Les matériaux que nous avons choisis comme exemples d'applications ont été limités au domaine des verres industriels et des verres d'oxydes mais cette méthode a également été très souvent appliquée à d'autres matériaux tels que les verres de chalcogénures, les alliages métalliques amorphes, les composés obtenus par évaporation sous vide.

\section{Bibliographie}

[1] Quasebart, K., Sprechsaal 49 (1916) 3.

[2] Tool, A. Q., EICHLIn, C. C., J. Opt. Soc. Am. 4 (1920) 340 ; J. Opt. Soc. Am. 8 (1924) 419.

[3] Rouquerol, J., BoIvinet, P., Differential thermal analysis (Academic Press) 2 (1972) 23.

[4] Wilburn, F. W., Thomasson, C. V., J. Soc. Glass Technol. 42 (1958) 206.

Wilburn, F. W., Thomasson, C. V., Cole, H., Symposium sur la fusion du verre. U. S. C. V. (1959) 373.

[5] OldField, L. F., Symposium sur la fusion du verre (1959) 383.

[6] Wilburn, F. W., Metcalfe, S. A., Warburton, R. S., Glass Technol. 6 (1965) 107.

[7] Bruneaux-Poulle, J., Defrain, A., Linh, N. T., J. Chim. Phys. 69 (1972) 76.
[8] LAmbert-Dusollier, G., Verres Réfract. 29 (1975) 65.

[9] Tool, A. Q., J. Am. Ceram. Soc. 31 (1948) 177.

[10] Broukal, J., Silikaty 3 (1959) 14.

[11] Kühne, K., Skatulla, W., Silikattechn. 10 (1959) 105.

[12] Mazurin, O. V., Streltsina, M. V., Totesh, A. S., Phys. Chem. Glasses 10 (1969) 63.

[13] Mazurin, O. V., Streltsina, M. V., J. Non-Crystal. Sol. 11 (1972) 199.

[14] ZarZyCKi, J., NAUdin, F., J. Non-Crystal. Sol. 5 (1971) 415.

[15] Prod’homme, M., Verres Réfract. 24 (1970) 151.

[16] Dusollier, G., Derobert, M., Verres Réfract. 23 (1969) 10.

[17] Kissinger, H. E., J. Res. Nat. Bur. Stand. 57 (1956) 217.

[18] Briggs, J., Carruthers, T. G., Phys. Chem. Glasses 17 (1976) 30. 